\title{
Efficiency Optimal of Inductive Power Transfer System Using the Genetic Algorithms
}

\author{
Jikun Zhou*, Rong Zhang, YiZhang \\ Institute of systems engineering, China academy engineering of physics, Mianyang, 621999 \\ email:zjikun@163.com
}

Keywords: IPT system, efficiency optimal, genetic algorithms

\begin{abstract}
The Inductive power transfer (IPT) systems' efficiency is determined by kinds of parameters. Aim at maximum the power transfer efficiency for Inductive power transfer (IPT) system, the efficiency of four basic topology IPT systems is analyzed. Based on the analysis, genetic algorithms (GA) are used to optimize IPT systems parameters to achieve the maximum of the power transfer efficiency. Before the GA optimization process, the IPT systems are compensated in a resonance condition which can achieve to minimize VA rating of the power supply and maximum power transfer. In the process of GA optimization, to ensure the final solutions achieving bifurcation-free operation, the solutions that generate bifurcation phenomenon (multi zero phase angle frequency) are excluded. The simulation results verify that the GA can find the best optimal solutions for all topology IPT systems.
\end{abstract}

\section{Introduction}

Inductive power Transfer (IPT) has been used in many commercial and industrial applications due to its ability to transfer electrical power without and physical contact [1-2]. The IPT system includes two independent mutually coupled part called primary and secondary respectively [4]. The primary part generates an essentially current $i_{\mathrm{p}}$ in the primary coil $L_{\mathrm{p}}$ with a resonant high frequency switching power supply. The primary compensation is used to minimize the VA rating of the supply. On the secondary part, compensation is also required to enhance the power transfer. A switched mode controller is normally used to control the power flow from the secondary coil $L_{\mathrm{s}}$ to the load.

Because of large air gaps via magnetic coupling, the efficiency is a signification target that must be considered when design of the IPT systems [6,7]. However, the systems' efficiency is determined by not only one parameter, such as air gap, operating frequency, coupling coefficient and so on, which makes the efficiency optimal design difficult $[9,10]$. To date, some analysis and optimal have been undertake. The effects of load and air gap variations are studied for different topology IPT systems [11].

However, the operating frequency effect is not considered. The reference [12] designs the IPT system by using an optimization algorithm. But the algorithm ignores the effect of coupling coefficient. Reference [13] analyzed all the parameters that influence the efficiency and presented an optimal algorithms design method, however, the algorithms cannot ensure to find out the most optimal solution and the bifurcation phenomenon is ignored.

In this paper, the GA is selected to efficiency optimal design of the IPT system undertakes the resonance conditions. In the process of the GA optimization, the solutions that generate bifurcation phenomenon are excluded. The simulation verifies that the GA can find the best optimal solutions for all base topology IPT systems.

\section{Efficiency analyses of base IPT systems}

With different compensation, there are four basic topology IPT systems that called SS, SP, PP, and PS are shown in Fig.1, where the first $S$ or $P$ stands for series or parallel LC compensation of primary resonance and the second $\mathrm{S}$ or $\mathrm{P}$ stands for series or parallel LC compensation of secondary resonance. The inductor $L_{\mathrm{p}}$ and $L_{\mathrm{s}}$ represent the primary and secondary coil respectively. The resistance $\mathrm{R}$ represents the load on the secondary, while the resistance $R_{\mathrm{p}}$ and $R_{\mathrm{S}}$ represent the 
self-resistance of $L_{\mathrm{p}}$ and Ls respectively.

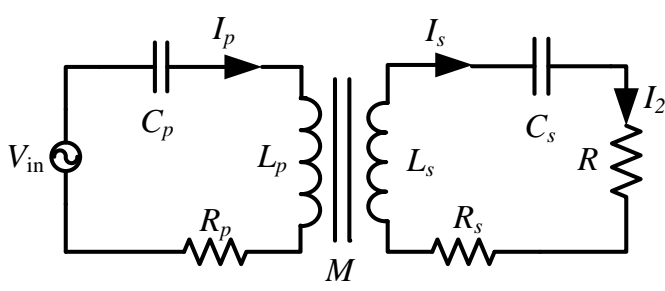

(a) SS

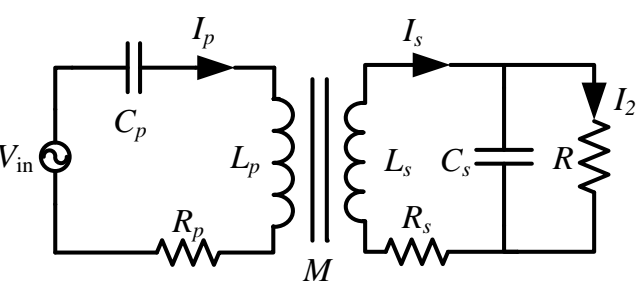

(b) SP

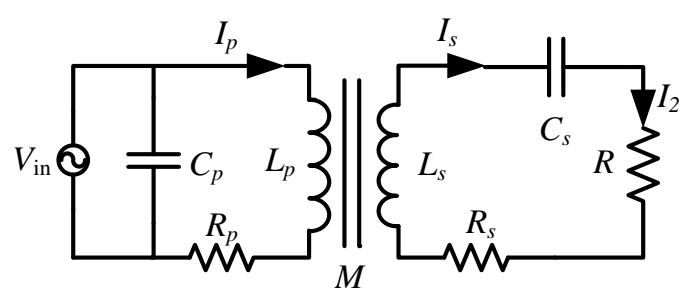

(c) PS

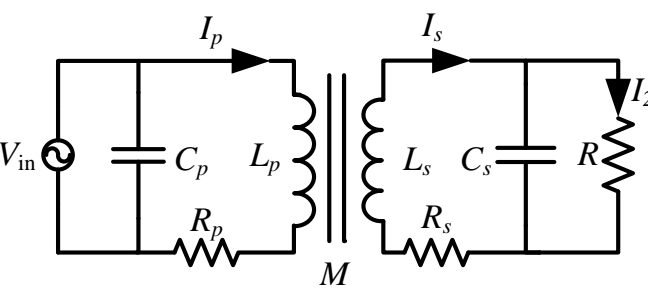

(d) PP

Fig.1 Four basic topologies of IPT system

The voltage $V_{\text {in }}$ is the output voltage of the converter. The impedance of the secondary $Z_{\mathrm{s}}$ whose value depends on the secondary compensation as given by

$$
Z_{s}= \begin{cases}j \omega L_{s}+\frac{1}{j \omega C_{s}}+R_{L} & \text { parallel secondary } \\ j \omega L_{s}+\frac{1}{j \omega C_{s}+1 / R_{L}} & \text { seris secondary }\end{cases}
$$

The reflected impedance $Z_{\mathrm{p}}$ is dependent on the transformer coupling and operating frequency, which is given by

$$
Z_{p}=\frac{\omega^{2} M^{2}}{Z_{s}}
$$

To achieve maximum power transfer, The capacitor $C_{\mathrm{s}}$ is chosen to compensate the large leakage inductances of the secondary coil $L_{\mathrm{s}}$ to achieve resonance conditions. Where, $\omega_{0}$ is the inherent frequency of the secondary part.

$$
C_{s}=\frac{1}{\omega_{0}^{2} L_{s}}
$$

The capacitor $C_{\mathrm{p}}$ is chosen to cancel the reactive part of the circuit seen by the source, to achieve maximum power transfer with minimum VA rating of the supply. With different topologies, the calculations of primary capacitor $C_{\mathrm{p}}$ for different topology IPT systems are shown in Table.1.

Table.1 Primary capacitor $C_{\mathrm{p}}$

\begin{tabular}{|c|c|c|c|}
\hline SS & $\frac{1}{\omega_{0}^{2} L_{p}}$ & PP & $\frac{L_{p}-M^{2} / L_{s}}{\left(\frac{M^{2} R}{L_{s}{ }^{2}}\right)^{2}+\omega_{0}{ }^{2}\left(L_{p}-M^{2} / L_{s}\right)^{2}}$ \\
\hline SP & $\frac{1}{\omega_{0}{ }^{2}\left(L_{p}-M^{2} / L_{s}\right)}$ & PS & $\frac{L_{p}}{\left(\frac{M^{2} R}{L_{s}^{2}}\right)^{2}+\omega_{0}{ }^{2} L_{p}{ }^{2}}$ \\
\hline
\end{tabular}

When operated at the secondary inherent frequency, the efficiency of the systems is given by [12]

$$
\eta_{p}=\frac{\eta_{s}=\frac{R}{\left(R+R_{s}\right)\left(1+\frac{R_{p}\left(R_{s}+R\right)}{\omega_{0} M^{2}}\right)}}{R+R_{s}+\frac{R_{s} R^{2}}{\omega_{0}^{2} L_{s}^{2}}+\frac{R_{p} R_{s}^{2}}{\omega_{0}^{2} M^{2}}+\frac{R}{\omega_{0}^{2} M^{2} L_{s}}\left(\omega_{0} L_{s}^{2}+\frac{R_{s} R}{\omega_{0}^{2}}\right)^{2}}
$$


Where

$$
M^{2}=k^{2} L_{p} L_{s}
$$

Where $k$ is coupling coefficient and subsistent (6) into (4) and (5), the systems efficiency can be given by

$$
\begin{gathered}
\eta_{s}=\frac{R \omega_{0}^{2} k^{2} L_{p} L_{s}}{\left(R+R_{s}\right)\left(\omega_{0}{ }^{2} k^{2} L_{p} L_{s}+R_{p} R_{s}+R_{p} R\right)} \\
\eta_{p}=\frac{R}{R+R_{s}+\frac{R_{s} R^{2}}{\omega_{0}^{2} L_{s}^{2}}+\frac{R_{p} R_{s}^{2}}{\omega_{0}^{2} k^{2} L_{p} L_{s}}+\frac{R}{\omega_{0}^{2} k^{2} L_{p} L_{s}^{2}}\left(\omega_{0} L_{s}^{2}+\frac{R_{s} R}{\omega_{0}}\right)^{2}}
\end{gathered}
$$

\section{Efficiency optimal with genetic algorithms}

When design the IPT systems, the efficiency usually is considered first. From (7) and (8), it can be seen that, four geometrical parameters, operate frequency $\omega_{0}$, primary inductor Lp, secondary inductor $L_{\mathrm{s}}$ and coupling coefficient $\mathrm{k}$ need to be optimal to achieve maximum power transfer. Therefore, the efficiency optimal problem is a maximization of the objective function with four geometrical parameters, which can be given by

$$
f_{o b j}= \begin{cases}\eta_{p}\left(\omega_{0}, L_{p}, L_{s}, k\right) & \text { parallel secondary } \\ \eta_{s}\left(\omega_{0}, L_{p}, L_{s}, k\right) & \text { seris secondary }\end{cases}
$$

However, it is difficult to find the optimal parameters with all of the four parameters with traditional numeric optimal algorithms. The genetic algorithms have become increasing important for this type of optimization problems by seeking the optimal solutions of the objective function with simulating the process of evolution and adaptation of living organisms.

The GA that presented by Holland is a computational optimization paradigm modeled on the concept of biological evolution [14]. It operates a population of random solutions to an optimization problem. A fitness function is used to evaluate the quality of all possible solutions. The solutions with high quality are selected to create the next generations.Therefore, the GA can be used to optimal the IPT systems by not using any complex mathematical information in the searching process.

During the optimal process of the IPT system, to ensure the zero phase angle frequency is equal to the resonance frequency, bifurcation phenomenal must be avoided. The necessary criteria is tabulated in Table.2.[5]

Table.2 Necessary criteria for bifurcation-free operation

\begin{tabular}{|c|c|}
\hline SS Topology & $\frac{\omega_{0} L_{s}}{R}<\sqrt{\frac{1}{2\left(1-\sqrt{1-k^{2}}\right)}}$ \\
\hline SP and PP Topologies & $\frac{R}{\omega_{0} L_{s}}<\frac{1}{k} \sqrt{1-k^{2}}$ \\
\hline PS Topology & $\frac{\omega_{0} L_{s}}{R}<\frac{1}{k}$ \\
\hline
\end{tabular}

The solutions that dissatisfy the necessary criteria should be eliminated. Moreover, the four dimensions $\omega_{0} 、 L_{\mathrm{p}} 、 L_{\mathrm{s}}$ and $\mathrm{k}$ also has max and minimize value, and are given by

$$
\left\{\begin{array}{c}
k_{-\min } \leq k \leq k_{-\max } \\
\omega_{0_{-} \min } \leq \omega_{0} \leq \omega_{0_{-} \max } \\
L_{p_{-} \min } \leq L_{p} \leq L_{p_{-} \max } \\
L_{s_{-} \min } \leq L_{s} \leq L_{s_{-} \max }
\end{array}\right\}
$$

The Process of GA optimal can be denotated as fllow.

Step1: Chromosone coding 
In this paper, static binary string is used to code the four dimensions. Assuming that the length of one dimension is $m$, the total length of the chromosome is $4 m$. The chromosome can be given by

$$
\begin{gathered}
X=\left[x_{4}, x_{3}, x_{2}, x_{1}\right]=\left[\omega_{0}, L_{p}, L_{s}, k\right] \\
x_{i}=b_{i^{*} m-1} b_{i^{*} m-2} \cdots b_{i^{*} m-m}
\end{gathered}
$$

The decimal value can be calculated by

$$
x_{i d}=x_{i_{-} \min }+\left(\sum_{j=i^{*} m-m}^{i^{*} m-1} b_{i^{*} m-j} 2^{j-1}\right) \frac{x_{i_{-} \max }-x_{i_{-} \min }}{2^{m}-1}
$$

Step2: Fitness evaluate

Creating an initial population of $\mathrm{N}$ individuals for evolution after chromosome coding was done. Then calculate the objective function value for every individual. To ensure the systems which has multi zero phase angle are not selected to copy and exist in next generation, the objective function should be divided by

$$
f_{o b j}^{\prime}\left(X_{j}\right)= \begin{cases}f_{o b j}\left(X_{j}\right) & \text { one zero phase angle } \\ 0 & \text { multi zero phase angle }\end{cases}
$$

Considering efficiency optimal is a maximization problem, the fitness function is equal to the objective function, and is give by

Step3: GA operator

$$
f_{\text {int ss }}\left(X_{j}\right)=f_{o b j}^{\prime}\left(X_{j}\right)
$$

The GA operator contains reproduction, crossover, and mutation operator. In this paper, roulette wheel selection is used in reproduction mechanism while single point crossover and single point mutation are used for crossover and mutation respectively. The efficiency optimal design of IPT system with GA is presented in Fig.2.

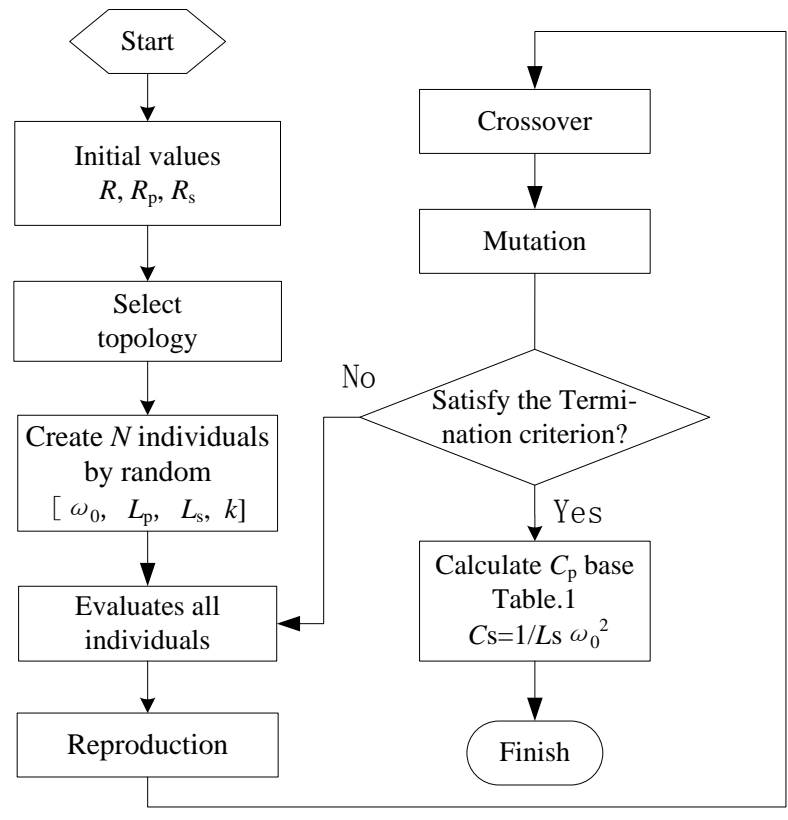

Fig. 2 Efficiency optimal result with GA

In the GA parameters, set the maximum number of generations $T=50$, the number of individuals $N=20$, the crossover probability $P_{\mathrm{c}}=0.6$, the mutation probability $P_{\mathrm{m}}=0.01$, the length of the chromosome $m=20$, and the four dimension are given by

$$
\left\{\begin{array}{c}
0.1 \leq k \leq 0.6 \\
30 \mu \mathrm{H} \leq L_{p} \leq 100 \mu \mathrm{H} \\
30 \mu \mathrm{H} \leq L_{s} \leq 100 \mu \mathrm{H} \\
2 \pi \times 15 \mathrm{kHz} \leq \omega_{0} \leq 2 \pi \times 50 \mathrm{kHz}
\end{array}\right\}
$$

The GA optimal process is shown in Fig. 3 and the final solutions are shown in Table. 4 


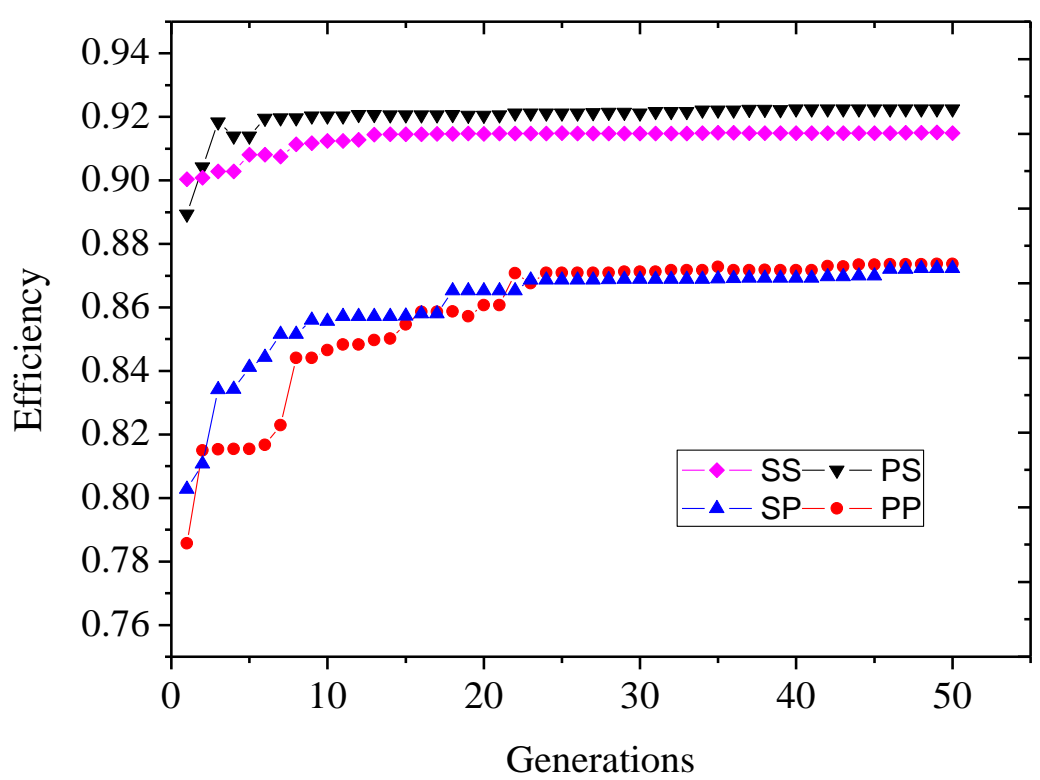

Fig.3 Efficiency optimal result with GA

Observation of Figure 3 reveals that the GA can find the best optimal solutions for all topology IPT systems. On the other hand, in the beginning of the process, not all the best solution in each population is being propagated to the subsequent generation. That's because the solutions with the highest efficiency may be not satisfy the necessary criteria of bifurcation-free.

Table.3 Optimized results of different topologies

\begin{tabular}{|l|l|l|l|l|}
\hline Parameter & SS & SP & PS & PP \\
\hline$L_{\mathrm{p}}(\mu \mathrm{H})$ & 99.94 & 95.58 & 99.36 & 99.75 \\
\hline$C_{\mathrm{p}}(\mu \mathrm{F})$ & 0.21 & 0.17 & 0.12 & 0.12 \\
\hline$L_{\mathrm{s}}(\mu \mathrm{H})$ & 73.59 & 69.98 & 60.99 & 98.35 \\
\hline$C_{\mathrm{s}}(\mu \mathrm{F})$ & 0.29 & 0.15 & 0.2 & 0.1 \\
\hline$k$ & 0.44 & 0.59 & 0.56 & 0.35 \\
\hline$f(\mathrm{kHz})$ & 34.74 & 48.86 & 45.38 & 49.83 \\
\hline$\eta(\%)$ & 91.49 & 87.23 & 92.25 & 87.36 \\
\hline
\end{tabular}

\section{Conclusion}

This paper has presented an optimization scheme based on the GA for the efficiency optimal design of the IPT system. Before the GA optimization process, the system is compensated in a resonance condition to minimize the VA rating of the power supply and achieve maximum power transfer. During the GA optimization process, the solutions that dissatisfy the necessary criteria should be eliminated to avoid bifurcation phenomenal occur in the finally solutions. The simulation verifies that the GA optimization can find the best optimal solutions for all topology IPT systems.

\section{References}

[1] Huang, Shyh-Jier;Lee, Tsong-Shing;Pai, Fu-Sheng;Huang, Tzyy-Haw. Method of feedback detection for loosely coupled inductive power transfer system with frequency-tracking mechanism. IEEE 10th International Conference on Power Electronics and Drive Systems (PEDS), 2013:784 787.

[2] Wu, Pengfei, Shi, Liming, Cai, Hua, Li, Yaohua. An inductively coupled power transfer system based power control for motor drives of rail transit vehicle. 17th International Conference on Electrical Machines and Systems, 2014: 202-205.

[3] J. T. Boys, G. A. Covic, and A. W. Green. Stability and control of inductively coupled power 
transfer systems. IEEE Proceedings Power Application. vol. 147, no. 1, 2000:37-43.

[4] B. Lee and K. Han. Proceedings of the Eighth International Conference on Electrical Machines and Systems.vol. 1, 2005:839-842.

[5] H.L. Li, A.P. Hu, G.A. Covic and C.S. Tang. Optimal coupling condition of IPT system for achieving maximum power transfer. electronics letters 1st January 2009, Vol. 45 No. 1

[6] Han, K.H., Lee, B.S., and Baek, S.H.: The design evaluation of inductive power-transformer for personal rapid transit by measuring impedance. Journal of Applied Physics, 2008: 103-105

[7] Moradewicz AJ, Kazmierkowski MP. Contactless energy transfer system with FPGA-controlled resonant converter. IEEE Transactions Industry Electronics 2010;57(9):3181 - 90.

[8] Sallan J, Villa JL, Llombart A, Sanz JF. Optimal design of ICPT systems applied toelectric vehicle battery charge. IEEE Transactions Industry Electronics, 2009;56(6):2140 - 9.

[9] Saeed Hasanzadeh, Sadegh Vaez-Zadeh. Efficiency analysis of contactless electrical power transmission systems. Energy Conversion and Management, 2013;65:487-496.

[10] Z. Michalewicz, Genetic Algorithms + Data Structures =Evolutionary Programs, Genetic Programming and Evolvable Machines, Springer, Berlin,1997: 263-306 